\author{
Filip Šuligoj \\ Bojan Jerbić \\ Bojan Šekoranja $\bowtie$ \\ Josip Vidaković \\ Marko Švaco
}

https://doi.org/10.21278/TOF.42203

ISSN 1333-1124

eISSN 1849-1391

\title{
INFLUENCE OF THE LOCALIZATION STRATEGY ON THE ACCURACY OF A NEUROSURGICAL ROBOT SYSTEM
}

\begin{abstract}
Summary
Precise navigation of surgical instruments is one of the most important features of autonomous surgical robots. In this paper, we introduce a concept of robot localization strategy and analyse its influence on the overall application error of a robot system for frameless stereotactic neurosurgery named RONNA. Localization strategies utilize specific angles at which the robot can approach a target point, orientations, and types of movement during the procedure of physical space fiducial marker localization and positioning to the target points. The localization strategies developed in this study are a neutral orientation strategy (NOS), an orientation correction strategy (OCS) and a joint displacement minimization strategy (JDMS). To evaluate the robot positioning performance with the localization strategies applied, we performed laboratory phantom measurements using a different number of fiducial markers in the registration procedure. When three, four, and five fiducial markers were used, the application error for the NOS was $1.571 \pm 0.256 \mathrm{~mm}$, $1.397 \pm 0.283 \mathrm{~mm}$, and $1.327 \pm 0.274 \mathrm{~mm}$, and for the OCS, it was $0.429 \pm 0.133 \mathrm{~mm}$, $0.284 \pm 0.068 \mathrm{~mm}$, and $0.260 \pm 0.076 \mathrm{~mm}$, respectively. The application error for the JDMS was $0.493 \pm 0.176 \mathrm{~mm}$ with four and $0.369 \pm 0.160 \mathrm{~mm}$ with five fiducial markers used.
\end{abstract}

Key words: $\quad$ medical robotics, localization strategy, accuracy, RONNA

\section{Introduction}

Robot-guided interventions (RGI) are used to accurately position or to navigate surgical instruments to specific targets planned by using medical images [1]. Medical images are derived using imaging technologies of computed tomography (CT), magnetic resonance imaging (MRI) or ultrasound; they are used to provide an insight into the interior of a human body. The main requirements imposed on a neurosurgical robot are precision and accuracy. Positioning accuracy of a neurosurgical robot is defined as a distance between the planned targets determined in the image space by the surgeon and the actual positions reached with a surgical instrument attached to the robot. Accuracy of a neurosurgical robot can be explained from three different aspects: intrinsic accuracy of the robot, registration accuracy, and application accuracy. The most relevant aspect, both for the surgeon and the patient, is the overall positioning accuracy, i.e. the application accuracy. 
Performance of a robot in regard to its positioning ability is determined by its repeatability and accuracy. Although repeatability, defined as the ability of the robot to return to the same position and orientation, is an important feature in medical robotics, absolute accuracy is more significant since the robot is sent to arbitrary target positions and orientations. Absolute accuracy of a robot is defined as its ability to move to a desired position in the threedimensional (3D) space with respect to a reference frame [2] [3]. Accuracy of a robot can also be defined as a measure of difference between the calculated position and the real position of the robot. In comparison to repeatability, the accuracy error is usually greater by an order of magnitude [4]. The positioning error of a robot results from the difference between the ideal kinematic model of the robot and the actual unit. Factors that cause robot errors are manufacturing and assembly imperfections, temperature influence on the dimensions and material characteristics of a part, backlash, and resolution of encoders [5]. The accuracy of a robot can be improved by using calibration methods. Research in the field of robot calibration focuses on various types of model optimization [6] [7] [8] and the development of measuring equipment and techniques used for calibration [9] [10]. Since the equipment for robot calibration can be expensive and the calibration procedure is a time-consuming task, in this study, we have used a robot with its nominal model (provided with a robot controller) to demonstrate that the application error of the robot system can be reduced without the use of calibration methods.

In order to provide a robot with the ability to navigate a surgical tool according to the trajectories planned in medical images, registration between the image space and the physical (robot) space is needed. In neurosurgical robotics, the patient registration implies geometrical transformations that align the view of a patient in medical images to the view of the patient in the robot coordinate system. Objects externally attached to the patient are called fiducial markers whose geometrical centres can be used as reference points for the "image-to-physical space" registration process. These reference points are called fiducial points. Fiducial localization is a process of determining the position of a fiducial point. For the registration to be successful, at least three corresponding fiducial points need to be localized in the coordinate system of the medical images and the coordinate system of the robot. Errors which occur in the localization procedure reduce the alignment accuracy in the registration process and have a negative effect on the registration accuracy. The patient localization can be based on different types of references (fiducials). Extrinsic methods rely on externally attached objects, such as bone-attached fiducial markers, skin-affixed fiducials, and frames, while intrinsic methods use the patient's anatomical landmarks [11]. Of all the above-mentioned methods, the method using bone-attached fiducial markers provides the highest degree of application and targeting accuracy. Bone-attached fiducial markers and rigid body registration are used with the RONNA system [12] and in this study. Major factors influencing the registration accuracy are fiducial marker type, the spatial distribution of fiducials, the number of fiducials used and the accuracy of the localization method. In the study [13], the idea of improving the target registration accuracy is proposed through the optimization of the distribution of fiducial points when the planned target trajectory is known. The study provides a practical approach for the surgeon to arrange the fiducial markers in a way that reduces the target registration error. Different approaches and performance metrics that can be used when planning the placement of fiducial markers are presented in [14] and [15]. Fitzpatrick et al. [16] show that a greater spread of fiducials leads to greater registration accuracy. In the study performed by Perwög et al. [17], it is shown that a greater number of fiducials used in registration had a positive influence on the accuracy of the computer-assisted navigation. This is one of the factors that will be tested for the robot system investigated in our study.

The objective of this study is to measure the influence of different physical space localization strategies of the robot on the accuracy of a neurosurgical robot system. The starting 
hypothesis is that the magnitude of the registration error and the robot intrinsic error can be reduced by utilizing specific angles at which the robot approaches the target points, orientations, and types of movement during the robot fiducial marker localization procedure and in positioning the robot tool at the planned target points. The RONNA robot system for frameless stereotactic neurosurgery, [18], [19], is used for testing the robot localization strategies. The localization strategies developed are the neutral orientation strategy (NOS), the orientation correction strategy (OCS), and the joint displacement minimization strategy (JDMS). To evaluate the robot application accuracy in three different approaches to the robot localization procedure, we performed laboratory phantom measurements using two different registration methods; three, four, and five fiducial markers were used in the registration procedure.

\section{Error analysis of a neurosurgical robot system}

The robot intrinsic accuracy and the registration accuracy are two major factors which generate the application accuracy of a neurosurgical robot system. Liu et al., [20], analysed and improved the application accuracy of Neuromaster, a 5 degrees of freedom (DOF) neurosurgical robot system. In their research, the focus was on improving the robot intrinsic accuracy through the use of neural networks to compensate for the joint transmitting error. The analysis of the robot positioning error in that research applies to ours.

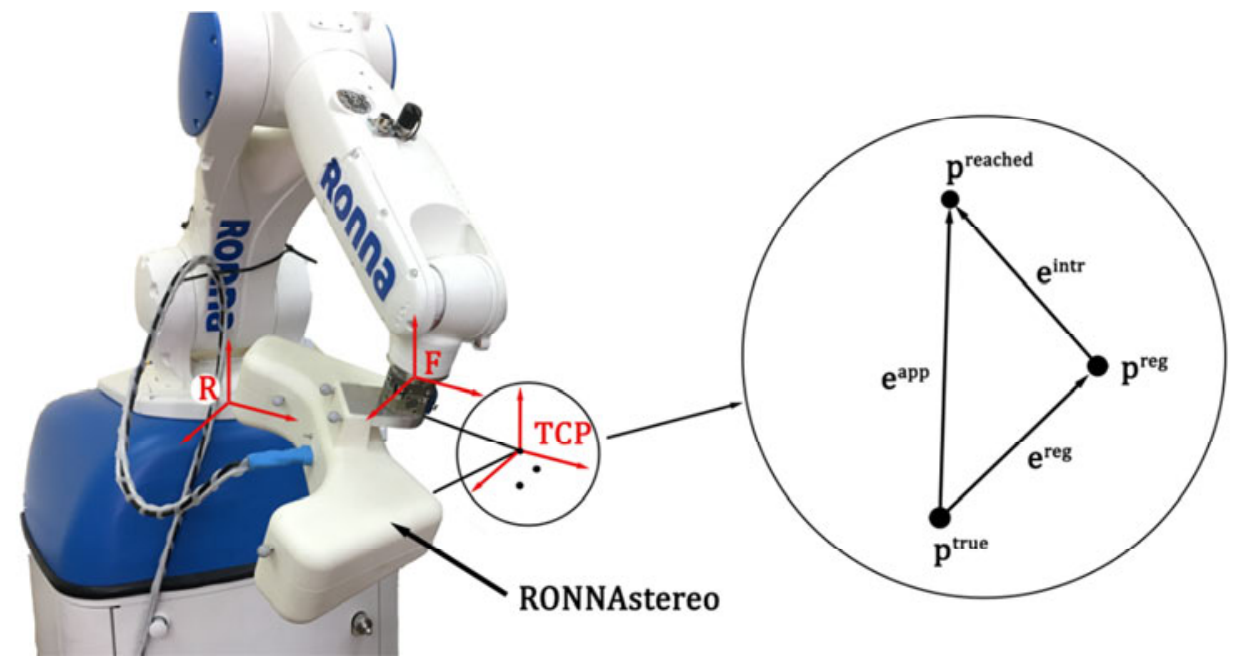

Fig. 1 Demonstration of the neurosurgical robot system positioning errors

The application error of the robot system, $\mathbf{e}^{\text {app }}$, regardless of the reference coordinate system, is a distance between the true position of the target, $\mathbf{p}^{\text {true }}$, and the actual position the robot tool has reached, $\mathbf{p}^{\text {reached }}$. Two infrared cameras with macro lenses (RONNAstereo) attached to the robot flange (Fig. 1) are used for the localization of fiducial markers and for measuring the application error. The magnitude of the robot system application error is given as a sum of the registration error, $\mathbf{e}^{\text {reg }}$, and the robot intrinsic error, $\mathbf{e}^{\mathrm{intr}}$ :

$$
\left\|\mathbf{e}^{\text {app }}\right\|=\left\|\mathbf{e}^{\text {reg }}+\mathbf{e}^{\mathrm{intr}}\right\| .
$$

The point $\mathbf{p}^{\text {reg }}$ is a point which is transformed from the image space to the physical space in the registration procedure; it can be defined as:

$$
\mathbf{p}^{\text {reg }}=\mathbf{p}^{\text {true }}+\mathbf{e}^{\text {reg }},
$$

where $\mathbf{e}^{\text {reg }}$ is the target registration error (TRE). The target registration error is defined as a distance between the planned image target location and the physical target location after the registration. The norm of the TRE can be estimated as [21] [22]: 


$$
\operatorname{TRE}^{2}(\mathbf{r}) \approx \frac{\mathrm{FLE}^{2}}{N}\left(1+\frac{\sum_{\mathrm{k}=1}^{3} \frac{\mathrm{d}_{k}^{2}}{\mathrm{f}_{k}^{2}}}{3}\right),
$$

with $N$ being the number of fiducial markers, $\mathrm{d}_{\mathrm{k}}$ the minimal distance of the target point $\mathbf{r}$ from the $k$-th principal axis, and $\mathrm{f}_{\mathrm{k}}$ the root mean square (RMS) distance of the fiducial markers from the $k$-th axis. The fiducial localization error (FLE) is defined as the Euclidean distance between the true and the measured distance of the fiducial marker location. The FLE is present in both the image and the physical space. The FLE in the image space is a result of noise produced by imaging artefacts, resolution of the reconstructed images produced by CT or an MRI scanner, and accuracy of the localization method. In our previous study, [23], we measured the image space FLE. When the developed localization algorithm was used, based on the ground truth estimation, the FLE was $0.162 \mathrm{~mm}$. For our robot system, the FLE in the physical space is a consequence of positioning errors of the robot during localization, calibration between the robot flange and RONNAstereo, resolution of the two cameras, and the algorithm used for calculating the centres of the fiducial markers. According to equation (3), the magnitude of TRE or $\mathbf{e}^{\text {reg }}$ depends on the number of fiducial markers, the spatial configuration of fiducial markers, the location of target points, and the FLE. In the study conducted by Siebold et al., [24], the TRE is used for the approximation of the safety margin between the drill tip and the nearby anatomical structures during the robotic bone milling task. The difference between the point where the robot is sent to, $\mathbf{p}^{\text {reg }}$, and the point that the tool tip reaches, $\mathbf{p}^{\text {reached }}$, is a result of the robot intrinsic error, $\mathbf{e}^{\text {intr. }}$

$$
\mathbf{p}^{\text {reached }}=\mathbf{p}^{\text {reg }}+\mathbf{e}^{\text {intr }} \text {. }
$$

The position of the robot is defined by six joint angles, $\boldsymbol{\theta}=\left(\theta_{1}, \theta_{2}, \theta_{3}, \theta_{4}, \theta_{5}, \theta_{6}\right.$, $)$, and a change in the robot tool position is determined by the change of the robot joint angles. The position of the robot tool in the Cartesian coordinate system can be calculated based on the robot kinematic model as:

$$
\underset{T C P}{R} A={ }_{1}^{R} A \cdot{ }_{2}^{1} A \cdot{ }_{3}^{2} A \cdot{ }_{4}^{3} A \cdot{ }_{5}^{4} A \cdot{ }_{F}^{5} A \cdot{ }_{T C P}^{F} A
$$

Concerning the differences between the robot kinematic model and the physical unit, we expect that bigger changes of robot joint angles or greater distances between points should generally result in a greater deviation of the real robot tool position from the position calculated based on its nominal kinematic model. From the perspective of the neurosurgical robot positioning accuracy, this means that if the target is further away from the fiducial markers or if the orientation of the trajectory differs from the orientation of the robot localizing the fiducial markers, then we could expect greater positioning errors. For our system, a standard robot kinematic model supplied with a robot controller was used. The transformation ${ }_{\text {TCPA }}^{\mathbf{F}} \mathbf{A}$ between the tool flange shown as a coordinate system $\mathbf{F}$ in Fig. 1 and the robot tool centre point was calibrated using the standard four-point method included in the robot standard controller options.

\section{Experiment elements and measurement workflow}

The measurement procedure is similar to the standard workflow of the RONNA system which is presented in [18] and is divided into the following steps:

A. A polymer laboratory phantom is prepared for CT scanning. The laboratory phantom dimensions are 250 × $250 \mathrm{~mm}$. Retro-reflective spheres shown in Fig. 2 a) are used as five 
fiducial markers denoted as $\mathbf{m}_{1}-\mathbf{m}_{5}$ and ten targets denoted as $\mathbf{t}_{1}-\mathbf{t}_{10}$. The fiducial marker $\mathbf{m}_{5}$ is also used as the target $\mathbf{t}_{10}$.

B. The laboratory phantom is scanned using a CT scanner (Somatom Emotion ${ }^{\circledR}$, Siemens, Erlangen, Germany) to obtain medical images. The CT scanner parameters are: bone kernel H70h (sharp), slice thickness $0.70 \mathrm{~mm}$, image matrix 512 x 512 pixels with a voxel size of $0.48 \times 0.48 \times 0.70 \mathrm{~mm}$.

C. Fiducial markers are localized in the phantom images. For fiducial marker localization, we use the automatic localization algorithm developed in [23]. The RONNAplan planning software is used to visualize the phantom and to define operation trajectories in the coordinate system CT of the CT scanner. Each operation trajectory is composed of two points, an entry point $\mathbf{e}$ and the surgery target point $\mathbf{t}$. The term "target pose" denoted as $\mathbf{T}$ is defined as the position of the target point and the orientation of the axis between the entry and target points. This definition of the target pose leaves the rotation around the entry-target axis as a free variable which is used in the localization strategies. Examples of a planned trajectory and five localized fiducial markers are shown in Fig. 2 b).

a)

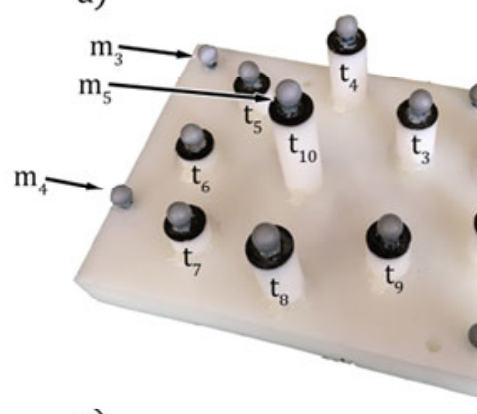

c)

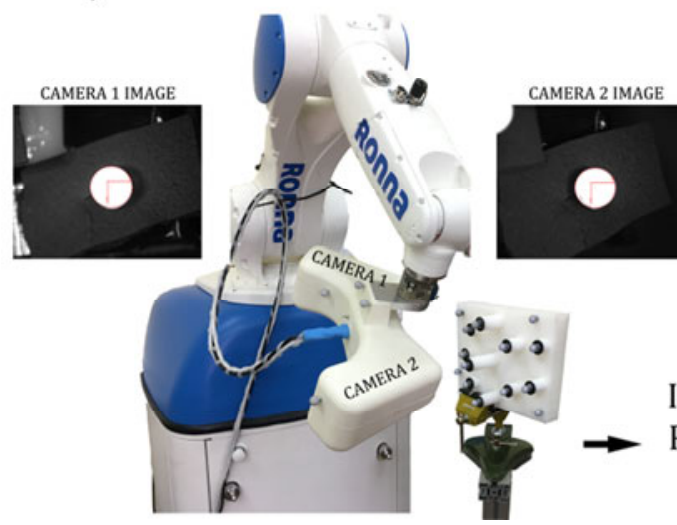

b)

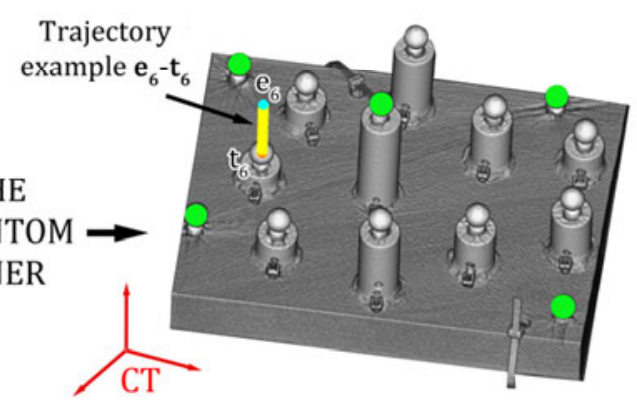

d)

IMAGE SPACE TO

PHYSICAL SPACE REGISTRATION
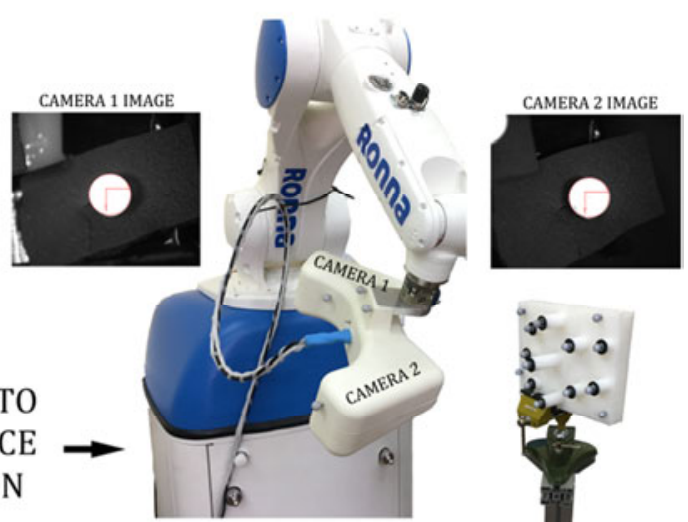

Fig. 2 Phases of the measurement procedure: a) laboratory phantom; b) laboratory phantom in the image space of the CT scanner with localized fiducial markers and an example of the defined trajectory; c) physical space fiducial marker localization using RONNAstereo; d) robot guided to the target pose to measure the application error

D. The phantom is fixed in the clamping device inside the work envelope of the robot as shown in Fig. 2 c). The optical tracking system (OTS) is used for the coarse localization of the fiducial markers in relation to the robot tool. The coarse localization is used for the initial guidance of the robot tool to the fiducial markers. RONNAstereo is then used for the precise localization of the fiducial markers in the robot coordinate system. The term "localization pose" denoted as $\mathbf{F}$ is defined as the position and the orientation of the robot tool when localizing a fiducial marker.

E. The registration between the CT scanner coordinate system CT and the robot base coordinate system $\mathbf{R}$ is performed after the localization. In this study, we apply two different registration techniques using three, four, and five fiducial markers, which will be 
further described in section 3.1, Registration methods. Three different localization strategies are used as elaborated in section 3.2, Robot localization strategies. The orientation correction strategy (OCS) and the joint displacement minimization strategy (JDMS) require re-localization with the calculated localization poses; therefore, steps D and $\mathrm{E}$ are repeated.

F. Operation trajectories are transformed into $\mathbf{R}$ and the robot tool is guided to each target pose as shown in Fig. 2 d). RONNAstereo is used to measure the Euclidean distance between the reached point and the true centre of the targeted retro-reflective sphere. In the measuring procedure, the magnitude of the application error of the robot system is measured in the coordinate system of RONNAstereo.

\subsection{Registration methods}

When the correspondence between points localized in the image space and points localized in the physical space is known, we use the point-based registration for finding their rigid transformation. To evaluate the impact of different registration methods and a different number of fiducial markers on the registration accuracy, in the measuring procedure, we perform registration with three, four, and five fiducial markers.

The rigid transformation $\mathbf{C T} \rightarrow \mathbf{R}$ in the case with three fiducial markers (which must be noncollinear and non-equidistant points is calculated by transforming the coordinates of the trajectory points into the coordinate system $\mathbf{M}$ of fiducial markers in both spaces. Coordinates of the three fiducial markers are used to form a transformation matrix ${ }_{\mathbf{M}}^{\mathbf{C O O R D}} \mathbf{A}$, where COORD can be either $\mathbf{R}$ or $\mathbf{C T}$. The rotation matrix is calculated from three unit vectors $(\mathbf{i}, \mathbf{j}$, and $\mathbf{k}$ ) calculated in equations (6) and the translation component of the homogeneous transformation matrix is used from the coordinates of the fiducial marker $\mathbf{m}_{1}$ :

$$
\begin{aligned}
& \mathbf{i}=\frac{\mathbf{m}_{2}-\mathbf{m}_{1}}{\left|\mathbf{m}_{2}-\mathbf{m}_{1}\right|}, \mathbf{k}=\frac{\mathbf{i} \times \frac{\mathbf{m}_{3}-\mathbf{m}_{1}}{\left|\mathbf{m}_{3}-\mathbf{m}_{1}\right|}}{\left|\mathbf{i} \times \frac{\mathbf{m}_{3}-\mathbf{m}_{1} \mid}{\left|\mathbf{m}_{3}-\mathbf{m}_{1}\right|}\right|}, \mathbf{j}=\frac{\mathbf{k} \times \mathbf{i}}{|\mathbf{k} \times \mathbf{i}|} \\
& \operatorname{cooRD}_{\mathbf{M}} \mathbf{A}=\left[\begin{array}{cccc}
\mathbf{i}_{\mathrm{x}} & \mathbf{j}_{\mathrm{x}} & \mathbf{k}_{\mathrm{x}} & \mathbf{m}_{1 \mathrm{x}} \\
\mathbf{i}_{\mathrm{y}} & \mathbf{j}_{\mathrm{y}} & \mathbf{k}_{\mathrm{y}} & \mathbf{m}_{1 \mathrm{y}} \\
\mathbf{i}_{\mathrm{z}} & \mathbf{j}_{\mathrm{z}} & \mathbf{k}_{\mathrm{z}} & \mathbf{m}_{1 \mathrm{z}} \\
0 & 0 & 0 & 1
\end{array}\right]
\end{aligned}
$$

Since both the image space and the physical space fiducial markers are localized, their coordinates are used for calculating two transformation matrices, ${ }_{\mathbf{M}}^{\mathbf{C T}} \mathbf{A}$ and ${ }_{\mathbf{M}}^{\mathbf{R}} \mathbf{A}$, by means of equations 6 and 7 . The coordinates of every trajectory point $\mathbf{e}$ or $\mathbf{t}$ planned in the $\mathbf{C T}$ coordinate system can be presented as ${ }_{\operatorname{tr}}^{\mathbf{C T}} \mathbf{A}$ and transformed to the robot coordinate system using the following equation:

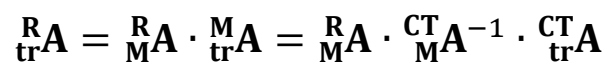

For calculating the rigid transformation $\mathbf{C T} \rightarrow \mathbf{R}$, when four or five fiducial markers are used, we implement the singular value decomposition (SVD). SVD is used for finding the rigid transformation that optimally aligns two sets of fiducial marker coordinates in the sense of least squares. For two sets of corresponding points, $\left\{\mathbf{c}_{1} \ldots \mathbf{c}_{\mathrm{i}}\right\}$ and $\left\{\mathbf{e}_{1} \ldots \mathbf{e}_{\mathrm{i}}\right\}$, the rotation matrix Rot and the translation vector $\mathbf{t}$ are calculated in a way that the following expression applies:

$$
(\operatorname{Rot}, \mathbf{t})=\min _{\operatorname{Rot} \in S O(d), t \in \operatorname{Rot}^{d}} \sum_{i=1}^{n}\left\|\left(\operatorname{Rot} \cdot \mathbf{c}_{\mathrm{i}}+\mathbf{t}\right)-\mathbf{e}_{\mathrm{i}}\right\|^{2}
$$


The complete procedure for the derivation of $\operatorname{Rot}$ and $\mathbf{t}$ and the mathematical proofs can be found in [25]. The transformation between the image space and the physical space is then given as:

$$
\underset{\mathrm{CT}}{\mathbf{R}} \mathrm{A}=\left[\begin{array}{cc}
\text { Rot } & \mathrm{t} \\
0 & 1
\end{array}\right]
$$

The coordinates of every trajectory point $\mathbf{e}_{\mathrm{i}}$ or $\mathbf{t}_{\mathrm{i}}$ planned in the CT coordinate system and denoted as ${ }_{\text {tr }}^{\mathbf{C T}} \mathbf{A}$ are transformed to the robot coordinate system using the following equation:

$$
\underset{\operatorname{tr}}{\mathbf{R}} \mathbf{A}=\underset{\mathrm{CT}}{\mathbf{R}} \mathbf{A} \cdot{ }_{\text {tr }}^{\mathbf{C T}} \mathbf{A}
$$

\subsection{Robot localization strategies}

Robots with six or more degrees of freedom can approach points in their workspace with different orientations and different joint configurations. If the geometrical properties and features of the fiducial markers allow localization at different angles, then the robot can use different orientations during localization. RONNA uses spherical fiducial markers which enable localization at arbitrary angles. Furthermore, each trajectory can be reached by the robot in numerous configurations, i.e. orientations around the longitudinal tool axis [26], as shown in Fig. 3. With the ability to approach fiducial markers at different angles during localization and the ability to change the angle around the x-axis in the target pose, we can implement different robot localization strategies.

Robot localization strategies have a goal of reducing errors in the patient registration procedure and in the positioning of the robot tool at the target pose. Every strategy uses a set $F$ which contains $n$ localization poses, $F=\left\{\mathbf{F}_{1}, \cdots, \mathbf{F}_{\mathrm{n}}\right\}$, and a set $T$ which contains $m$ target poses, $T=\left\{\mathbf{T}_{1}, \cdots, \mathbf{T}_{\mathrm{m}}\right\}$. As shown in Fig. 3 a), the position of the robot tool is determined by six joint states $\boldsymbol{\theta}$. The robot tool pose is defined using a Cartesian coordinate system translation vector $(\mathrm{x}, \mathrm{y}, \mathrm{z})$ and three orientation variables $(\alpha, \beta, \gamma)$ as a combination of Euler's angles with the Z-Y-X convention. In Fig. 3 a), the robot tool pose is the transformation $\mathbf{R} \rightarrow \mathbf{T C P}$.
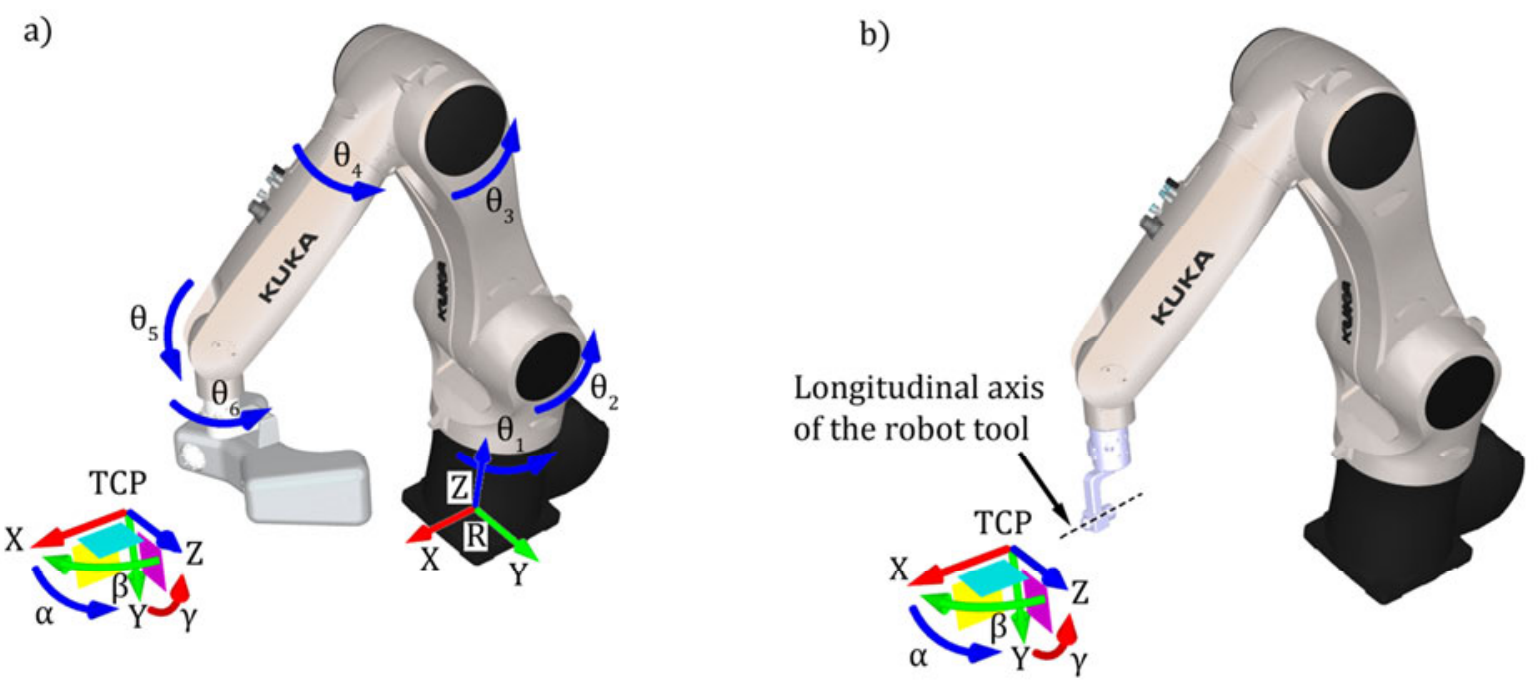

Fig. 3 Parameters defining a robot pose, a)Robot with RONNA stereo mounted; b)Robot with a surgical tool mounted; the tool uses the same virtual TCP as the RONNA stereo

In the following subsections, we present three localization strategies: a) Neutral orientation strategy, b) Orientation correction strategy, and c) Joint displacement minimization strategy. 


\subsubsection{Neutral orientation strategy (NOS)}

NOS is the simplest strategy in which the robot localizes each fiducial marker with the identical orientation of the tool, which is unrelated to the trajectory pose. During the localization procedure, the robot is positioned to the $n$ localization poses, each defined by the coordinates of a fiducial marker with identical robot tool orientation $\left(\alpha_{0}, \beta_{0}, \gamma_{0}\right)$ :

$$
\forall \mathrm{i} \in \mathrm{F}: \alpha_{\mathrm{i}}=\alpha_{0} ; \beta_{\mathrm{i}}=\beta_{0} ; \gamma_{\mathrm{i}}=\gamma_{0}
$$

Precise localization is accomplished by moving the robot to the centre of the two images captured with the RONNAstereo. Calibrated centres of the images from RONNAstereo cameras correspond to the physical tool centre point of the robot, as shown in Fig. 3 a) and b). It should be noted that during the fine localization, the robot is moved by linear movement commands until the centre of each image is reached. This is the reason why the backlash error is present in the localization procedure. This procedure provides positions of fiducial markers in R. Fiducial markers localized in the physical space are used for the registration with the image space. All entry and target points are transformed to the coordinate system of the robot; the robot tool orientation for every target pose is calculated from them in a way that the $\mathrm{x}$-axis of the robot tool is parallel with the vector between the entry and the target, and the value of free angle variable $\gamma$ is set to $-90^{\circ}$ with respect to the robot base (perpendicular to the horizontal plane).

\subsubsection{Orientation correction strategy (OCS)}

The initial part of OCS is the same as in NOS. The robot is positioned to the coordinates of every fiducial marker localized by the OTS using the identical initial robot tool orientation. Localization is accomplished in the same manner as in NOS. The position and orientation of each target pose is calculated after the initial localization. The orientation correction strategy then expands the neutral orientation strategy by adding an extra step. The value of free variable $\gamma$ is set to $-90^{\circ}$ with respect to the robot base for each calculated target pose. The robot re-localizes all of the $n$ fiducial markers with the orientation of the robot tool which is identical to the target pose orientation for each of $m$ trajectories, as shown in equation (13).

$$
\forall \mathrm{i} \in \mathrm{F}, \forall \mathrm{k} \in \mathrm{T}: \alpha_{\mathrm{i}, \mathrm{k}}=\alpha_{\mathrm{k}} ; \beta_{\mathrm{i}, \mathrm{k}}=\beta_{\mathrm{k}} ; \gamma_{\mathrm{i}, \mathrm{k}}=-90^{\circ}
$$

After re-localization, the registration is repeated and new target poses are calculated.

\subsubsection{Joint displacement minimization strategy (JDMS)}

JDMS uses the same procedure as NOS to obtain the initial position of each fiducial marker and target pose. From the initial coordinates of fiducial markers and target poses, JDMS calculates different robot joint configurations for the re-localization of each fiducial marker with respect to each target pose. Robot joint configurations are calculated in a way that the sum of differences of $\boldsymbol{\theta}$ between the localization pose of every fiducial marker and the target pose for each set of $n$ fiducial markers and $m$ trajectories is minimized, as shown in equation (14):

$$
\begin{aligned}
& \forall \mathrm{k} \in \mathrm{T}, \forall \gamma_{T} \in\left\{\gamma_{0}+5^{\circ} * \mathrm{j}: \mathrm{j} \in \mathbb{Z} ;-6 \leq \mathrm{j} \leq 6\right\}, \\
& \forall \mathrm{i} \in \mathrm{F}, \forall \alpha \in\left\{\alpha_{0}+10^{\circ} * \mathrm{l}: \mathrm{l} \in \mathbb{Z} ;-3 \leq \mathrm{l} \leq 3\right\}, \\
& \forall \beta \in\left\{\beta_{0}+10^{\circ} * \mathrm{o}: \mathrm{o} \in \mathbb{Z} ;-3 \leq \mathrm{o} \leq 3\right\}, \\
& \forall \gamma \in\left\{\gamma_{0}+10^{\circ} * \mathrm{p}: \mathrm{p} \in \mathbb{Z} ;-3 \leq \mathrm{p} \leq 3\right\}: \min \left|\theta_{\mathrm{k}, \gamma_{T}}-\theta_{i, \alpha, \beta, \gamma}\right|
\end{aligned}
$$

The localization procedure is repeated with the calculated localization poses using joint movement commands for joint angles denoted as $\boldsymbol{\theta}_{\boldsymbol{F}}$. Approach points are used before moving the robot tool to the localization poses for the purpose of removing potential backlash errors. 
The approach point for every localization pose is defined as the function of robot joint angles at the localization pose $\boldsymbol{\theta}_{\text {APPROACH}}=\boldsymbol{\theta}_{\boldsymbol{F}}-\delta, \delta=1^{\circ}$. After re-localization, the registration is repeated with the new points and the target poses are calculated. Before the robot tool is positioned to the target pose denoted as $\boldsymbol{\theta}_{T}$, it is first sent to the approach point denoted as $\boldsymbol{\theta}_{T}-\delta$, again to remove a potential influence of the backlash.

\section{Experiment results}

In the experiments, we used a CT image of a laboratory phantom and the same localized coordinates of five fiducial markers and ten retro-reflective spheres which were used as target points. All markers were localized following the measurement procedure described in section 3 and using the strategies elaborated in section 3.2. Eight series of measurements were performed, one for each position of the phantom in the robot workspace. In each measurement series, the robot localization was performed separately for each localization strategy and each number of fiducial markers used for registration (three, four, and five).

Based on eight laboratory phantom positions and ten target points for each phantom position, the average application errors for individual targets and the overall strategies were calculated and shown in Tables 1, 2, and 3. For NOS and the registration with three, four, and five fiducial markers, the total average error was $1.571 \pm 0.256 \mathrm{~mm}, 1.397 \pm 0.283 \mathrm{~mm}$, and $1.327 \pm 0.274 \mathrm{~mm}$, respectively. NOS localizes the fiducial markers with the identical neutral orientation and uses the same $\gamma$ angle when positioning the robot tool to the target pose. Due to these features, a large difference in orientation is possible between the robot localization pose and the robot target pose. The result is a potentially bigger registration error, a bigger robot intrinsic error and, consequently, a bigger application error.

Table 1 Neutral orientation strategy measurement results

Individual trajectory average error

\begin{tabular}{cc|cccccccccc} 
& & $\mathrm{t}_{1}$ & $\mathrm{t}_{2}$ & $\mathrm{t}_{3}$ & $\mathrm{t}_{4}$ & $\mathrm{t}_{5}$ & $\mathrm{t}_{6}$ & $\mathrm{t}_{7}$ & $\mathrm{t}_{8}$ & $\mathrm{t}_{9}$ & $\mathrm{t}_{10}$ \\
\hline No of points & 3 & 1.645 & 1.665 & 1.925 & 1.586 & 1.705 & 1.378 & 1.374 & 1.383 & 1.444 & 1.602 \\
used in the & 4 & 1.545 & 1.600 & 1.752 & 1.325 & 1.506 & 1.179 & 1.166 & 1.228 & 1.294 & 1.378 \\
registration & 5 & 1.466 & 1.533 & 1.667 & 1.244 & 1.452 & 1.120 & 1.095 & 1.174 & 1.233 & 1.291 \\
\hline
\end{tabular}

Overall error

\begin{tabular}{ccc} 
Average & Max & Min \\
\hline $\mathbf{1 . 5 7 1}$ & 2.245 & 1.081 \\
$\mathbf{1 . 3 9 7}$ & 2.105 & 0.804 \\
$\mathbf{1 . 3 2 7}$ & 2.007 & 0.770 \\
\hline
\end{tabular}

The overall average application error shown in Table 2 for OCS and the registration with three, four, and five fiducial markers was $0.429 \pm 0.133 \mathrm{~mm}, 0.284 \pm 0.068 \mathrm{~mm}$ and $0.260 \pm 0.076$ $\mathrm{mm}$, respectively. OCS uses the same orientation when re-localizing the fiducial markers for every trajectory and when moving the robot tool to the target pose. The result of this approach is a smaller registration error and a smaller robot positioning error. Since the orientation of the robot tool does not change during the entire procedure, we can state with certainty that the errors in the calibration of the robot tool do not influence the registration error or the robot positioning error. The backlash error should be present in both the fiducial marker localization and the positioning at the target pose, the same as in the NOS. The OCS does not provide the optimum solution for either the registration or the robot positioning accuracy, but both are affected in a way that results in reduced errors.

Table 2 Orientation correction strategy measurement results

Individual trajectory average error

\begin{tabular}{cc|cccccccccc} 
& & $\mathrm{t}_{1}$ & $\mathrm{t}_{2}$ & $\mathrm{t}_{3}$ & $\mathrm{t}_{4}$ & $\mathrm{t}_{5}$ & $\mathrm{t}_{6}$ & $\mathrm{t}_{7}$ & $\mathrm{t}_{8}$ & $\mathrm{t}_{9}$ & $\mathrm{t}_{10}$ \\
\hline No of points & 3 & 0.346 & 0.380 & 0.616 & 0.556 & 0.338 & 0.289 & 0.388 & 0.320 & 0.473 & 0.583 \\
used in the & 4 & 0.238 & 0.296 & 0.401 & 0.231 & 0.271 & 0.367 & 0.224 & 0.232 & 0.310 & 0.275 \\
registration & 5 & 0.210 & 0.260 & 0.338 & 0.167 & 0.292 & 0.397 & 0.231 & 0.221 & 0.265 & 0.222 \\
\hline
\end{tabular}

Overall error

\begin{tabular}{ccc} 
Average & Max & Min \\
\hline $\mathbf{0 . 4 2 9}$ & 0.773 & 0.201 \\
$\mathbf{0 . 2 8 4}$ & 0.486 & 0.153 \\
$\mathbf{0 . 2 6 0}$ & 0.442 & 0.128 \\
\hline
\end{tabular}


The overall average application error (shown in Table 3) for JDMS and the registration with four and five fiducial markers was $0.493 \pm 0.176 \mathrm{~mm}$ and $0.369 \pm 0.160 \mathrm{~mm}$, respectively. JDMS uses different orientations when re-localizing every fiducial marker for every target pose and also calculates and uses different angles of the $\gamma$ robot tool angle in the target pose. This approach should reduce the robot positioning error since the function calculates the orientation with which the minimal joint movement is necessary for movements between the localization poses and each target pose. Furthermore, every localization pose and target pose are approached from the same joint direction to remove the influence of backlash. Because the localization of every fiducial marker is performed with a different robot tool orientation, the potential errors in the calibration of the robot tool have a significant influence on the registration error.

Table 3 Joint displacement minimization strategy measurement results

\begin{tabular}{|c|c|c|c|c|c|c|c|c|c|c|c|c|c|c|}
\hline & & \multicolumn{10}{|c|}{ Individual trajectory average error } & \multicolumn{3}{|c|}{ Overall error } \\
\hline & & $t_{1}$ & $t_{2}$ & $\mathrm{t}_{3}$ & $\mathrm{t}_{4}$ & $\mathrm{t}_{5}$ & $\mathrm{t}_{6}$ & $\mathrm{t}_{7}$ & $\mathrm{t}_{8}$ & $\mathrm{t}_{9}$ & $t_{10}$ & Average & Max & Min \\
\hline No of points & 4 & 0.353 & 0.308 & 0.705 & 0.283 & 0.363 & 0.543 & 0.628 & 0.482 & 0.546 & 0.713 & 0.493 & 0.834 & 0.194 \\
\hline registration & 5 & 0.254 & 0.247 & 0.575 & 0.151 & 0.323 & 0.310 & 0.517 & 0.372 & 0.393 & 0.548 & 0.369 & 0.691 & 0.016 \\
\hline
\end{tabular}

The box plot in Fig. 4 shows the measured application errors for all localization strategies and all numbers of fiducial markers used in the registration process. OCS showed the smallest average application error followed by JDMS. As expected, NOS had the biggest application error. For every localization strategy, the average application error was smaller if a larger number of fiducial markers had been used in the registration. For OCS and JDMS, the data was more closely distributed when a larger number of fiducial markers were used, while NOS had the smallest distribution when only three fiducial markers were used.

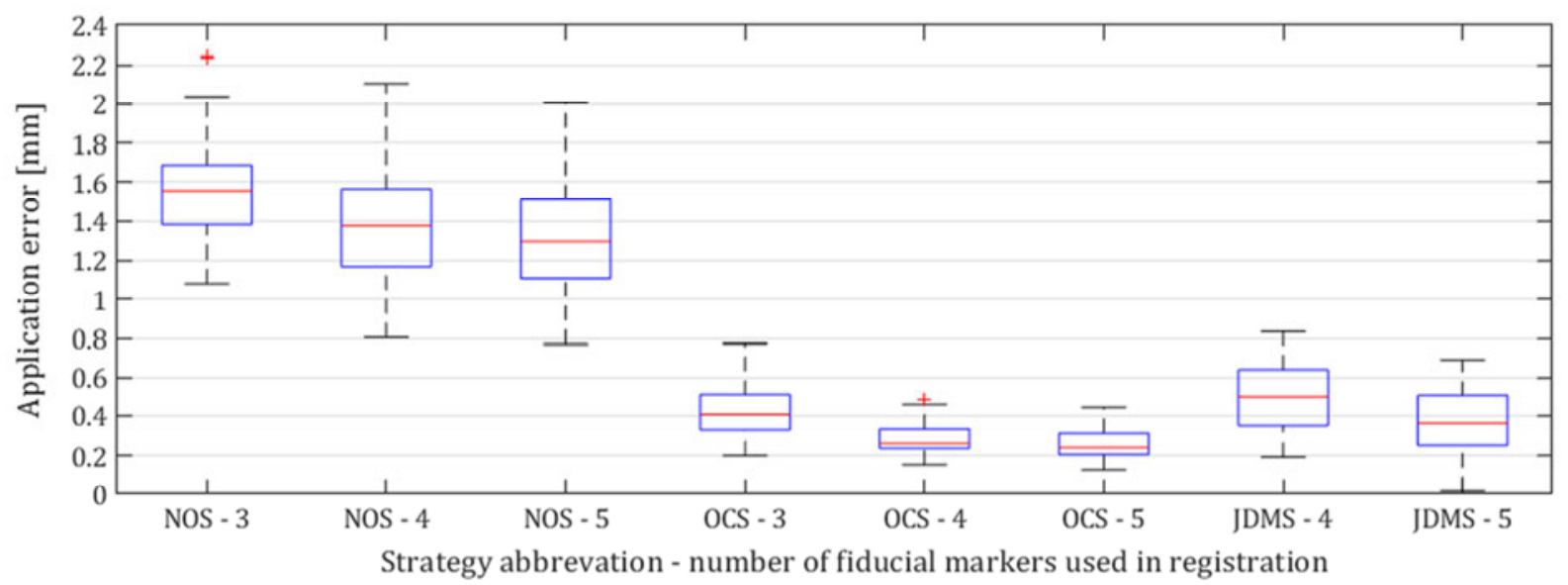

Fig. 4 Measurement results for all localization strategies

It should be noted that the conducted laboratory measurements do not consider several factors that can occur in in vivo procedures, such as irregularities of the surgical instruments, brain shift, patient movement in the fixation clamp, changes in the patient anatomy between imaging and operating, and motion artefacts in the CT scans.

\section{Conclusion}

In this study, we have introduced and tested the influence of three different robot localization strategies on the application accuracy of a neurosurgical robot system. The experimental results have shown that the impact of the robot localization strategy on the overall application accuracy of the neurosurgical robot system is significant. When comparing 
the localization strategies with the most accurate (OCS) and the least accurate result (NOS), the application error is smaller by $72.69 \%, 79.65 \%$ and $80.39 \%$ for the registrations using three, four, and five fiducial markers, respectively. Based on the overall measurement results we can conclude that a larger number of fiducial markers used in the registration procedure improves the accuracy of a surgical robot system for every of the three robot localization strategies. In general, the measurement results in this study enable us to get a good insight into the application accuracy of the RONNA system and into a possibility of estimating the expected error when positioning the robot tool in respect of the type of markers, number of fiducial markers, localization method, and localization strategies. The presented localization strategies can be used with any 6DOF (or 7DOF) revolute robot used in neurosurgical procedures to significantly reduce the application error regardless of the fact whether the robot has been previously calibrated or used with the nominal kinematic model supplied with the factory robot controller.

In future research, we plan to develop a new strategy as a combination of a modified and an improved JDMS, which should outperform OCS. The strategy will be implemented with a calibrated model of the robot to reduce errors caused by the robot tool calibration and to obtain even smaller application errors. In order to operate in the robot's optimal workspace region, the problem of robot base placement with respect to target points can strongly influence the dexterity of an articulated robotic arm. In our future research, robot localization strategies will be combined with position planning strategies which search for the optimal placement of a neurosurgical robot [27].

\section{Acknowledgement}

The authors would like to acknowledge the support of the European Regional Development Fund received through the project RONNA (robotic neuronavigation) and of the Croatian Scientific Foundation received through the research project ACRON (HRZZ-4192). The authors would also like to thank the entire team from the University Hospital Dubrava and especially Assistant Professor Darko Chudy and Dr Domagoj Dlaka, for the tremendous help in all medical aspects.

\section{REFERENCES}

[1] A. Hussain, A. Malik, M. U. Halim, and A. M. Ali, "The use of robotics in surgery: a review," International Journal of Clinical Practice, vol. 68, no. 11, pp. 1376-1382, Nov. 2014, https://doi.org/10.1111/ijcp.12492.

[2] P. S. Shiakolas, K. L. Conrad, and T. C. Yih, "On the Accuracy, Repeatability, and Degree of Influence of Kinematics Parameters for Industrial Robots," International Journal of Modelling and Simulation, vol. 22, no. 4, pp. 245-254, Jan. 2002, https://doi.org/10.1080/02286203.2002.11442246.

[3] Albert Nubiola, "Contribution to improving the accuracy of serial robots," Montreal, 2014.

[4] Y. H. Andrew Liou, P. P. Lin, R. R. Lindeke, and H. D. Chiang, "Tolerance specification of robot kinematic parameters using an experimental design technique-the Taguchi method," Robotics and Computer-Integrated Manufacturing, vol. 10, no. 3, pp. 199-207, Jun. 1993, https://doi.org/10.1016/0736-5845(93)90055-O.

[5] C. Mavroidis, S. Dubowsky, P. Drouet, J. Hintersteiner, and J. Flanz, "A systematic error analysis of robotic manipulators: application to a high performance medical robot," 1997, vol. 2, pp. 980-985.

[6] A. Nubiola and I. A. Bonev, "Absolute robot calibration with a single telescoping ballbar", Precision Engineering, vol. 38, no. 3, pp. 472-480, Jul. 2014, https://doi.org/10.1016/j.precisioneng.2014.01.001.

[7] L. Ma, P. Bazzoli, P. M. Sammons, R. G. Landers, and D. A. Bristow, "Modeling and calibration of high-order jointdependent kinematic errors for industrial robots," Robotics and Computer-Integrated Manufacturing, vol. 50, pp. 153167, Apr. 2018, https://doi.org/10.1016/j.rcim.2017.09.006.

[8] M. Švaco, B. Šekoranja, F. Šuligoj, and B. Jerbić, "Calibration of an Industrial Robot Using a Stereo Vision System," Procedia Engineering, vol. 69, pp. 459-463, 2014, https://doi.org/10.1016/j.proeng.2014.03.012.

[9] Y. Meng and H. Zhuang, "Self-Calibration of Camera-Equipped Robot Manipulators," The International Journal of Robotics Research, vol. 20, no. 11, pp. 909-921, Nov. 2001, https://doi.org/10.1177/02783640122068182.

[10] F. Boochs, R. Schutze, C. Simon, F. Marzani, H. Wirth, and J. Meier, "Increasing the accuracy of untaught robot positions by means of a multi-camera system," 2010, pp. 1-9. 
[11] G. Eggers, J. Mühling, and R. Marmulla, "Image-to-patient registration techniques in head surgery,” International Journal of Oral and Maxillofacial Surgery, vol. 35, no. 12, pp. 1081-1095, Dec. 2006, https://doi.org/10.1016/j.ijom.2006.09.015.

[12] D. Dlaka et al., "Brain biopsy performed with the RONNA G3 system: a case study on using a novel robotic navigation device for stereotactic neurosurgery," The International Journal of Medical Robotics and Computer Assisted Surgery, p. 1884, Dec. 2017, https://doi.org/10.1002/rcs.1884.

[13] M. Wang and Z. Song, "Improving target registration accuracy in image-guided neurosurgery by optimizing the distribution of fiducial points," The International Journal of Medical Robotics and Computer Assisted Surgery, vol. 5, no. 1, pp. 26-31, Mar. 2009, https://doi.org/10.1002/rcs.227.

[14] R. R. Shamir, L. Joskowicz, and Y. Shoshan, "Fiducial Optimization for Minimal Target Registration Error in ImageGuided Neurosurgery," IEEE Transactions on Medical Imaging, vol. 31, no. 3, pp. 725-737, Mar. 2012, https://doi.org/10.1109/TMI.2011.2175939.

[15] M. Franaszek and G. S. Cheok, "Selection of fiducial locations and performance metrics for point-based rigid-body registration", Precision Engineering, vol. 47, pp. 362-374, Jan. 2017, https://doi.org/10.1016/j.precisioneng.2016.09.010.

[16] J. M. Fitzpatrick, "The role of registration in accurate surgical guidance," Proceedings of the Institution of Mechanical Engineers, Part H: Journal of Engineering in Medicine, vol. 224, no. 5, pp. 607-622, May 2010, https://doi.org/10.1243/09544119JEIM589.

[17] M. Perwög, Z. Bardosi, and W. Freysinger, "Experimental validation of predicted application accuracies for computer-assisted (CAS) intraoperative navigation with paired-point registration", International Journal of Computer Assisted Radiology and Surgery, Aug. 2017, https://doi.org/10.1007/s11548-017-1653-y.

[18] M. Švaco, B. Šekoranja, F. Šuligoj, J. Vidaković, B. Jerbić, and D. Chudy, “A Novel Robotic Neuronavigation System: RONNA G3," Strojniški vestnik - Journal of Mechanical Engineering, vol. 63, no. 12, Dec. 2017, https://doi.org/10.5545/sv-jme.2017.4649.

[19] B. Jerbić, G. Nikolić, D. Chudy, M. Švaco, and B. Šekoranja, "Robotic application in neurosurgery using intelligent visual and haptic interaction," International Journal of Simulation Modelling, vol. 14, no. 1, pp. 71-84, 2015, https://doi.org/10.2507/IJSIMM14(1)7.290.

[20] J. Liu, Y. Zhang, and Z. Li, "Improving the Positioning Accuracy of a Neurosurgical Robot System," IEEE/ASME Transactions on Mechatronics, vol. 12, no. 5, pp. 527-533, Oct. 2007, https://doi.org/10.1109/TMECH.2007.905694.

[21] J. M. Fitzpatrick, J. B. West, and C. R. Maurer, "Predicting error in rigid-body point-based registration," IEEE transactions on medical imaging, vol. 17, no. 5, pp. 694-702, 1998.

[22] J. M. Fitzpatrick and J. B. West, "The distribution of target registration error in rigid-body point-based registration," IEEE transactions on medical imaging, vol. 20, no. 9, pp. 917-927, 2001.

[23] F. Šuligoj, M. Švaco, B. Jerbić, B. Šekoranja, and J. Vidaković, “Automated Marker Localization in the Planning Phase of Robotic Neurosurgery," IEEE Access, vol. 5, pp. 12265-12274, 2017, https://doi.org/10.1109/ACCESS.2017.2718621.

[24] M. A. Siebold, N. P. Dillon, R. J. Webster, and J. M. Fitzpatrick, "Incorporating target registration error into robotic bone milling," 2015, p. 94150R.

[25] O. Sorkine-Hornung, M. Rabinovich, and Department of Computer Science, ETH Zurich, "Least-Squares Rigid Motion Using SVD,” p. 5, Jan. 2017.

[26] M. Švaco, P. Koren, B. Jerbić, J. Vidaković, B. Šekoranja, and F. Šuligoj, "Validation of Three KUKA Agilus Robots for Application in Neurosurgery," in Advances in Service and Industrial Robotics, vol. 49, C. Ferraresi and G. Quaglia, Eds. Cham: Springer International Publishing, 2018, pp. 996-1006.

[27] J. Vidaković, B. Jerbić, M. Švaco, F. Šuligoj, and B. Šekoranja, "Position planning for collaborating robots and its application in neurosurgery," Tehnicki vjesnik - Technical Gazette, vol. 24, no. 6, Dec. 2017, https://doi.org/10.17559/TV-20170213110534.

Submitted:

05.4 .2018

Accepted

09.5.2018
Filip Šuligoj

Bojan Jerbić

Bojan Šekoranja

bojan.sekoranja@fsb.hr

Josip Vidaković

Marko Švaco

Department of Robotics and Production

System Automation, Faculty of

Mechanical Engineering and Naval

Architecture, University of Zagreb,

Zagreb, Croatia 